\title{
The AMS technique as an important tool for the measurement of astrophysi- cal cross sections
}

\author{
Luis Acosta ${ }^{1, *}$, Paulina Amador-Valenzuela ${ }^{2}$, Eduardo Andrade ${ }^{1}$, Libertad Barrón-Palos ${ }^{1}$, David Belmont ${ }^{1}$, Luis E. \\ Charón ${ }^{1}$, Efraín Chávez ${ }^{1}$, Hilarion $S$. Cruz-Galindo ${ }^{2}$, Jorge García-Ramírez ${ }^{1}$, David Godos-Valencia ${ }^{1}$, Arcadio Huerta ${ }^{1}$, \\ Daniel J. Marín-Lámbarri ${ }^{1}$, Josselin N. Martínez ${ }^{3}$, Javier Mas-Ruiz ${ }^{1}$, Carmen G. Méndez ${ }^{1,4}$, Eliud Moreno ${ }^{2}$, Ghiraldo \\ Murillo $^{2}$, Santiago Padilla ${ }^{1}$, Rafael Policroniades ${ }^{2}$, Roberto Raya-Arredondo ${ }^{2}$, Guadalupe Reza ${ }^{1}$, María Rodríguez- \\ Ceja $^{1}$, Ebanibaldo Sánchez-Zúñiga ${ }^{1}$, Sinuhé Sandoval-Hipólito ${ }^{1}$, Vijay R. Sharma ${ }^{1}$, Corina Solís ${ }^{1}$, Armando Varela ${ }^{2}$, \\ Pedro Villaseñor ${ }^{2}$, and Amacalli B. Zunun-Torres ${ }^{1}$
}

\author{
${ }^{1}$ Instituto de Física, Universidad Nacional Autónoma de México, Mexico City, Mexico \\ ${ }^{2}$ Instituto Nacional de Investigaciones Nucleares, Ocoyoacac, Mexico \\ ${ }^{3}$ Colegio Ciudad de México, Mexico City, Mexico. \\ ${ }^{4}$ CONACYT, Instituto de Física, Universidad Nacional Autónoma de México, Mexico City, Mexico
}

\begin{abstract}
Accelerator Mass Spectrometry is a technique commonly used to approach low concentrations of certain long half-life radioisotopes. The most important contribution of the technique is the accurate measure of organic sample ages, by separating masses 12,13 and 14 in the case of carbon allocated in such samples. However, the reach of AMS could cover many other scientific scopes, since it can give us a precise measure of a very small concentration of a radioisotope. On this direction, AMS can be used to approach reactions of interest for astrophysics, if we spot an specific radioisotope which concentration can be measure with AMS Starting with this, we have selected specific reactions involving ${ }^{14} \mathrm{C},{ }^{10} \mathrm{Be}$ and ${ }^{26} \mathrm{Al}$, produced with slow neutrons from a reactor and positive ions at an accelerator. The main idea is to produce a particular reaction and later to measure the radioisotopic concentration using AMS. In this study our first results for ${ }^{14} \mathrm{C}$ and ${ }^{10} \mathrm{Be}$ nuclei produced with neutrons, and the preliminary results for ${ }^{26} \mathrm{Al}$ nuclei produced with deuterium are shown.
\end{abstract}

\section{Introduction}

Most of times, to approach a good measurement of an specific nuclear reaction, particularly those important for astrophysics, is a difficult task. The energy range, the specific nuclei and the low cross sections are typical problems which currently appear for this kind of experimental studies. Underground accelerators, compact accelerator-based neutron sources and indirect techniques are the main solutions to develop an important amount of interesting reactions. Several studies have been carried out using these kind of methods [1-9].

Nevertheless it is always important to find alternative solutions, considering the big importance of the understanding of certain reactions with difficult access and essential for a better description of stellar and primordial nucleosynthesis. On this direction, we started 5 years ago a campaign of measurements to test the capabilities of the Accelerator Mass Spectrometry technique (AMS) as a possible tool to approach specific reactions in a direct way. The proposal of this technique starts with the fact that it may give an accurate value of the radioisotopic concentration contained in a certain sample; if such radioisotope is a product of an interesting reaction, this concentration is then directly related to the total cross section of the reaction selected.

*e-mail: acosta@fisica.unam.mx
The main constrain of the use of AMS as a technique to find a particular reaction cross section is that it can be used only for a number of radioisotopes, which main characteristic is to have a long half-life. Some examples are ${ }^{10} \mathrm{Be}$, ${ }^{14} \mathrm{C},{ }^{26} \mathrm{Al},{ }^{36} \mathrm{Cl}$ and ${ }^{129} \mathrm{I}$ radioisotopes, whose half-lifes are among the thousands and millions of years. Another important issue to consider when AMS is used, is to have a good radiochemical protocol to isolate the correct compound that can be studied with this technique. This important step is probably the main key to have a good and precise measurement.

An aspect not directly related to the AMS technique, but the first step to start this kind of studies, is the reaction production. We have to find the way to produce the reaction that we are planning to study. This implies to choose a target, a projectile and an energy range. The projectile may be produced, as usual, using some kind of particle accelerator. A good alternative studied in this work, is to use thermal neutrons from a research reactor. On the other hand, the targets must be carefully chosen, considering they have to be suitable to be radiochemically processed to achieve the isolation of the final compound which can be studied with AMS.

In the following sections the different reactions studied, the facilities used and the results achieved will be described. 


\section{Candidate reactions}

To select the possible reactions feasible to be studied with AMS, we have to base them in the radioisotopes that can be measured in our AMS facility. ${ }^{10} \mathrm{Be},{ }^{14} \mathrm{C}$ and ${ }^{26} \mathrm{Al}$ are radioisotopes well characterized and previously measured in our Mass Spectrometer. On that way, the ${ }^{14} \mathrm{~N}(\mathrm{n}, \mathrm{p}){ }^{14} \mathrm{C}$ reaction is a feasible one, if we consider it can be produced with thermal neutrons. Its cross section is large and well known, hence it is a good candidate reaction to test the technique proposed $[10,11]$.

Another reaction involving thermal neutrons which is just known by indirect measurements in the ${ }^{9} \mathrm{Be}(\mathrm{n}, \gamma){ }^{10} \mathrm{Be}$ reaction. This later reaction is important to complete for instance the knowledge regarding the primordial and star synthesis of light elements [12, 13].

In the case of possible reactions produced with beam particles we found two interesting ones involving ${ }^{26} \mathrm{Al}$ : the ${ }^{28} \mathrm{Si}(\mathrm{d}, \alpha){ }^{26} \mathrm{Al}$ reaction at low energies could be a possible answer to the anomalous amount of ${ }^{26} \mathrm{Mg}$ found in meteorites [14]; on the other hand the ${ }^{25} \mathrm{Mg}(\mathrm{p}, \gamma)^{26} \mathrm{Al}$ is one of the important reactions in the stellar evolution, and the production of ${ }^{26} \mathrm{Al}$ found with the existing models still disagree the ${ }^{26} \mathrm{Al}$ observed by its gamma decay $[15,16]$.

\section{Facilities}

There is a tradition of 40 years in the Ion Beam Analysis studies by using the different Mexican accelerators. In that sense, for our present studies it was easy to find low energy beams with high intensities. That is the case of the 5.5 MV Van de Graaff Accelerator [17] (Fig 1 left) of the Carlos Graef Fernández Laboratory (Physics Institute, UNAM) and the $6 \mathrm{MV}$ Tandem Van de Graaff Accelerator [18] (Fig 1 center) of the National Institute of Nuclear Research (ININ). Both facilities may produces high intensity deuterium beams at energies from 0.8 till 4.5 MeV. These beams were used to produce our ${ }^{26} \mathrm{Al}$ reactions.

On the other hand, we started to explore the possibilities to use the TRIGA Mark III (Fig 1 right) research reactor [19] also at ININ, which is currently used just for chemical studies and analytical techniques using neutrons. The low energy neutrons $(\sim 25 \mathrm{meV})$ produced by this facility (fluxes of thermal neutrons of up to $3.3 \times 10^{13} \mathrm{n} / \mathrm{cm}^{2} \mathrm{~s}$ ) are suitable to approach astrophysical neutron capture reactions. Starting with this, we tested the ${ }^{10} \mathrm{Be}$ and ${ }^{14} \mathrm{C}$ reactions chosen for our studies using the facility described.

For the AMS measurement we used the 1 MV Tandetron accelerator of the Accelerator Mass Spectrometry National Laboratory (LEMA, for their meaning in Spanish) of the Physics Institute, UNAM. This is the most recent accelerator in Mexico (it was inaugurated on 2013) and is well calibrated to discriminate radionuclei from its simile stable ones in a ratio of $1 \times 10^{-15}$ [20]. This high precision makes to LEMA an ideal tool to explore reactions with small cross sections.

\section{Targets}

The target selection is probably the most important thing for this kind of studies, considering they have to be used as cathodes (to use AMS technique), once the reaction was produced. For our first attempts to produce ${ }^{26} \mathrm{Al}$ with deuterium beams we use a combination of silicon and aluminum powder (50-50), inserted directly in one of the cylinders which are used as part of a cathode for the AMS system. Unfortunately, as it has shown in the RBS spectrum of the Fig. 3a, the area facing the beam is small enough to loss most of the beam (which is mostly hitting on copper, the material of the cylindrical container) $[21,22]$. This problem was solved a couple of years later, by using a silicon wafer target with a size 10 times larger than the LEMA cylinder used as cathode (see spectrum of Fig. 3b). It is important to mention that this solution implies a radiochemical separation, which was not consider in the first test. Nevertheless, the radiochemical process to disintegrate the silicon wafer was previously tested and proved, finding totally acceptable results.

On the other hand, for thermal neutron reactions the following target were used: For the ${ }^{14} \mathrm{C}$ reaction we used uracil $\left(\mathrm{C}_{4} \mathrm{H}_{4} \mathrm{~N}_{2} \mathrm{O}_{2}\right)$, a well-known material with a good amount of nitrogen, enough for our experimental proposal; in the case of ${ }^{10} \mathrm{Be}$ reaction, $\mathrm{BeO}$ samples were chemically prepared separating this compound from $8 \mathrm{ml}$ of Be standard solution (Beryllium ICP Standard Solution, $\mathrm{Be}_{4} \mathrm{O}\left(\mathrm{C}_{2} \mathrm{H}_{3} \mathrm{O}_{2}\right)_{6}, 1000 \mathrm{mg} / \mathrm{l} \mathrm{Be}$ Merck), which is currently used either as carrier or blank in AMS studies.

\section{Experimental results.}

\section{$5.1{ }^{28} \mathrm{Si}(\mathrm{d}, \alpha)^{26} \mathrm{Al}$ reaction.}

In our first attempt to produce ${ }^{26} \mathrm{Al}$ by means of ${ }^{28} \mathrm{Si}(\mathrm{d}, \alpha){ }^{26} \mathrm{Al}$ reaction, we used deuterium beams at different energies starting from $1 \mathrm{MeV}$ till $2 \mathrm{MeV}$. Considering we used a copper cylinder with a small area as target, we lost around $80 \%$ of the beam for most of the samples. This situation taken us to measure extremely small total cross sections (of the order of $\mu \mathrm{b}$ ), making questionable our methodology (a more detailed description can be consulted in $[21,22])$.

For such reason we decided to make a second test, but this time using a silicon wafer as target, starting from $1 \mathrm{MeV}$ till $4.8 \mathrm{MeV}$ deuterium energies. The results of this new measurement were very promising, considering we achieved much larger ${ }^{26} \mathrm{Al}$ currents, once AMS was employed. The plot of the Fig. 4 shows a very stable ${ }^{26} \mathrm{Al} /{ }^{27} \mathrm{Al}$ ratio of around $5.9 \times 10^{-10}$, at least three orders of magnitud larger that in the first measurement. The same figure shows one of the $\Delta \mathrm{E}-\mathrm{E}$ spectra obtained with these samples, in which a clean and well-defined spot of ${ }^{26} \mathrm{Al}$ counts can be observed. A deep description of this preliminar study can be found in [23].

In spite the good results achieved in this second attempt, some modifications to our setup are necessary, considering the measure of the beam current impinging the 


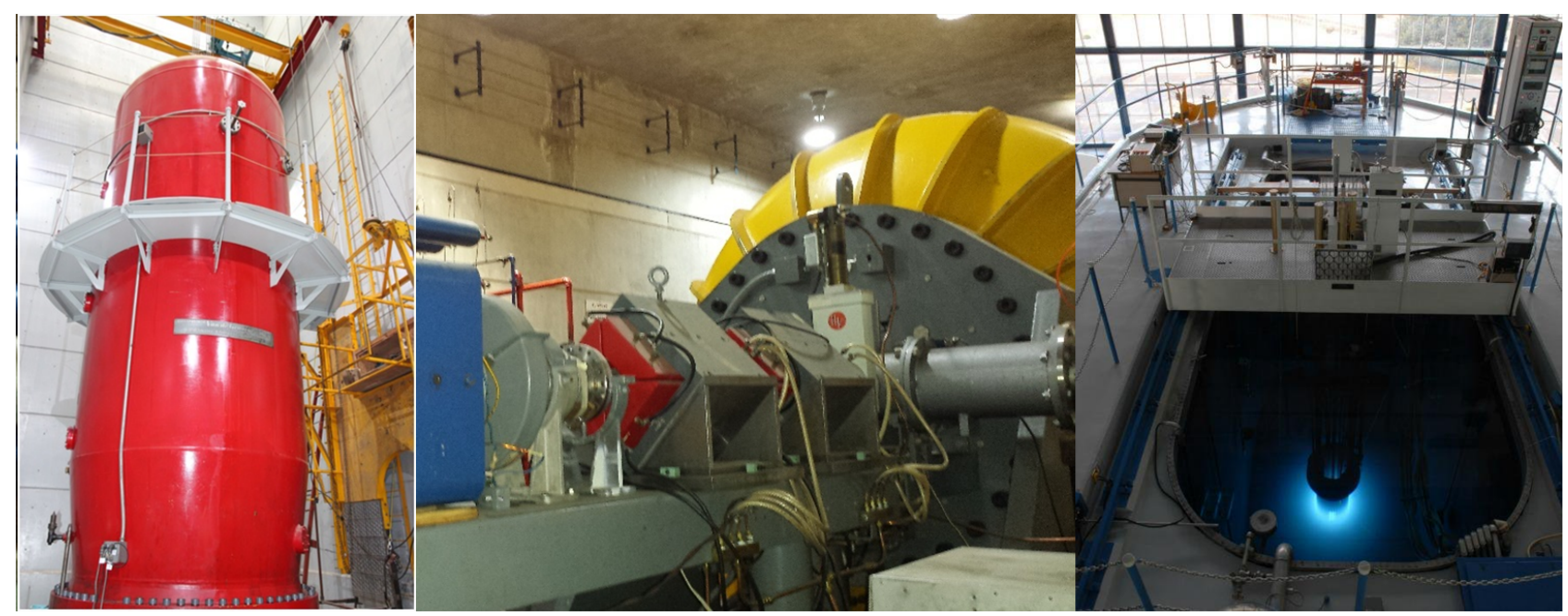

Figure 1. Mexican facilities used for the irradiation stage of the present studies. Left: 5.5 MV Van de Graaff Accelerator, Carlos Graef Fernández Laboratory, IFUNAM, Mexico. Center: 6 MV Tandem Van de Graaff Accelerator and Right: TRIGA Mark III Research reactor, both from the National Institute of Nuclear Research, Mexico.

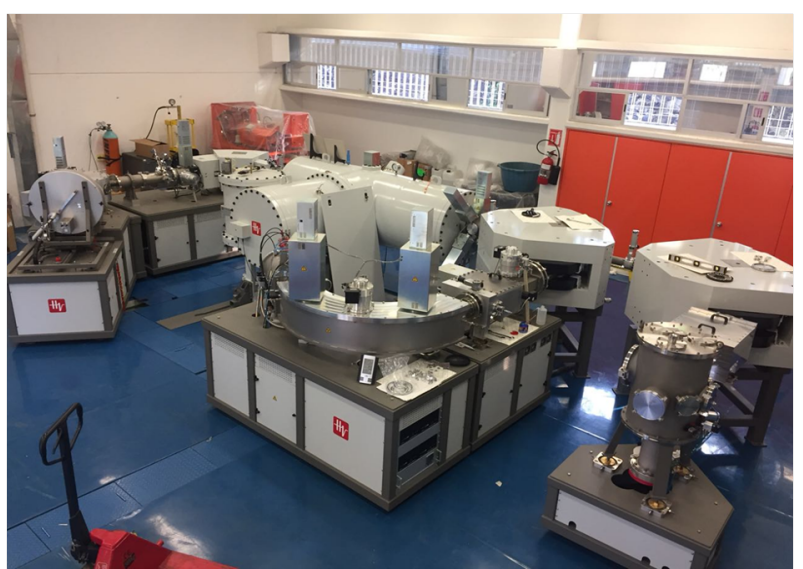

Figure 2. 1 MV Tandetron of the Accelerator Mass Spectrometry National Laboratory, LEMA IFUNAM, Mexico, which was used for the AMS stage of the present studies.

target has to be achieved with a better precision, since we observed instabilities during the data taking. A most precise measurement is programmed in the coming months.

\section{$5.2{ }^{14} \mathrm{~N}(\mathrm{n}, \mathrm{p}){ }^{14} \mathrm{C}$ reaction.}

In order to test a good protocol for thermal neutron capture reaction measurements using the technique proposed, the ${ }^{14} \mathrm{~N}(\mathrm{n}, \mathrm{p}){ }^{14} \mathrm{C}$ reaction was produced in the TRIGA III research reactor before mentioned, using the highest flux of thermal neutrons for 20 seconds in two different samples (targets) of uracil. An example of the $\Delta \mathrm{E}-\mathrm{E}$ spectra achieved with AMS is shown in Fig. 5, where the ${ }^{14} \mathrm{C}$ events can be well separated from other beams produced from the sample irradiated. After the analysis of the ${ }^{14} \mathrm{C}$ concentrations obtained, our data gave a total cross section for ${ }^{14} \mathrm{~N}(\mathrm{n}, \mathrm{p}){ }^{14} \mathrm{C}$ of $2.07 \pm 0.37 \mathrm{~b}$, which is in good agreement with other results reported in the literature [10,11], demonstrating the effectiveness of the method used. A more detailed description of this measurement can be consulted in [24].

\section{$5.3{ }^{9} \mathrm{Be}(\mathrm{n}, \gamma){ }^{10} \mathrm{Be}$ reaction.}

Regarding ${ }^{10} \mathrm{Be}$ reaction, it was also produced by using thermal neutrons from the TRIGA III research reactor. In this case a thermal neutron flux of $2.3 \times 10^{13} \mathrm{n} / \mathrm{cm}^{2} \mathrm{~s}$ in $\mathrm{BeO}$ samples was used for periods of 30 minutes and 2 hours. Fig. 6 shows a typical $\Delta$ E-E spectrum obtained with the AMS system for the $\mathrm{BeO}$ samples irradiated. In the spectrum is easy to observe by eye, the separation among ${ }^{10} \mathrm{Be}$ and ${ }^{10} \mathrm{~B}$, its isobar. Once the analysis of the data achieved was performed, we obtained a value for the total cross section of the ${ }^{9} \mathrm{Be}(\mathrm{n}, \gamma){ }^{10} \mathrm{Be}$ reaction of $9.7 \pm 0.53 \mathrm{mb}$. This value is in acceptable agreement with previous measurements found in the literature [11,25], nevertheless it is important to remark that ours is the first direct measurement reported. This last aspect makes even more valuable the technique used, considering the possibility to obtain direct measurements for complicated reactions. More details of this study can be consulted in [24].

\section{Conclusions and perspectives.}

Taking advantages of low energy accelerators, a research reactor and a AMS system, all of them Mexican facilities, we could develop a campaign of measurements of different nuclear reactions, most of them important in astrophysics and not previously measured in a direct way. The method has three stages: sample irradiation, radiochemical preparation and AMS analysis. The final result of this combination may decant in the measure of a total cross section of the initial reaction selected.

We presented in this work a review of our first measurements regarding ${ }^{28} \mathrm{Si}(\mathrm{d}, \alpha){ }^{26} \mathrm{Al},{ }^{14} \mathrm{~N}(\mathrm{n}, \mathrm{p}){ }^{14} \mathrm{C}$ and ${ }^{9} \mathrm{Be}(\mathrm{n}, \gamma){ }^{10} \mathrm{Be}$ reactions. The first case is still in progress 

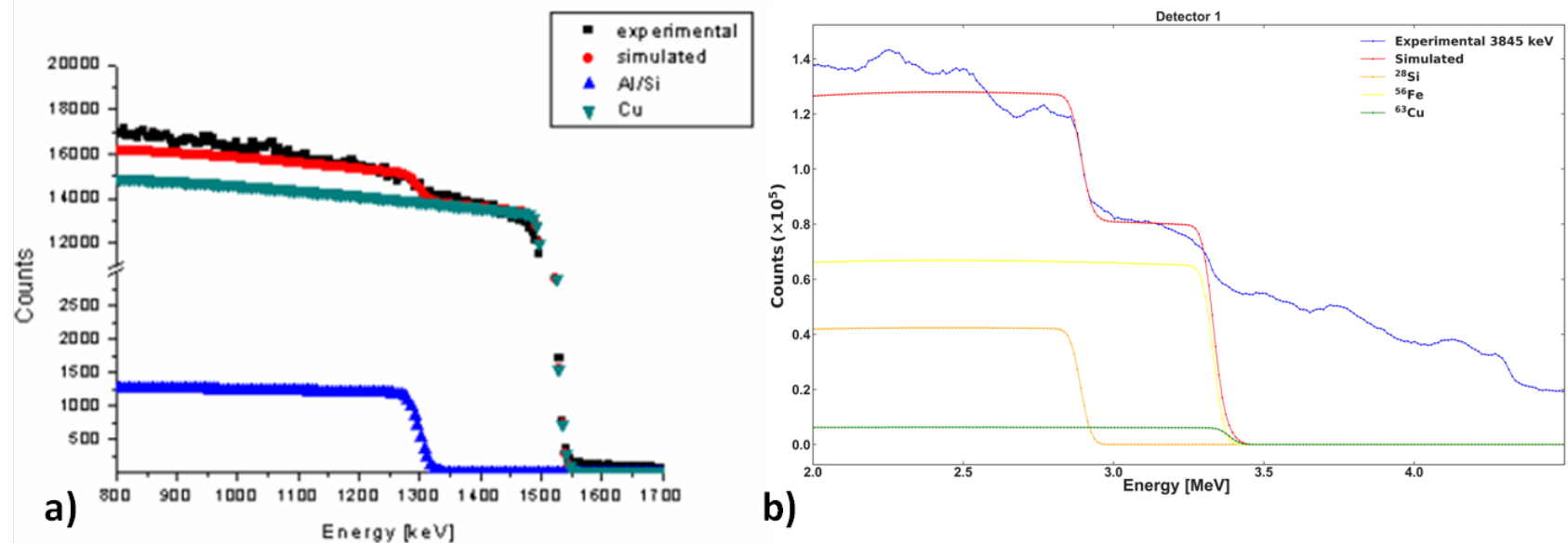

Figure 3. Rutherford Back Scattering (RBS) spectra for two different approaches to the ${ }^{26} \mathrm{Al}$ reaction. The spectra show the different components where the deuterium beam is hitting. In (a) we use a small size cylinder facing the beam, then most of the beam was scattered by copper, which explains the large step of $\mathrm{Cu}$ in the spectrum (taken from [22]). A better situation is presented in (b) where a larger Si target was used. In this last, the beam scattered by silicon is much more notorious, indicating that most of the beam is been used to produce the reaction of interest.
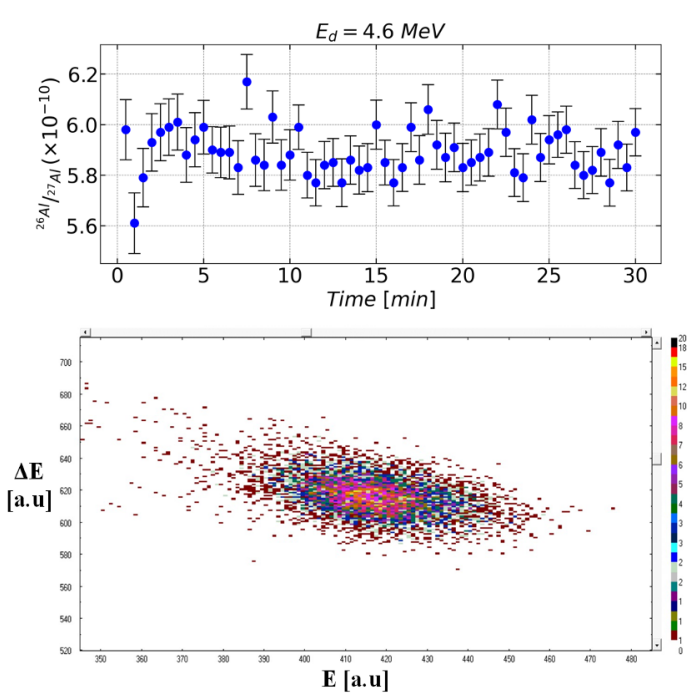

Figure 4. Some results achieved for the counting of ${ }^{26} \mathrm{Al}$ using AMS. On top the ${ }^{26} \mathrm{Al} /{ }^{27} \mathrm{Al}$ ratio for $4.6 \mathrm{MeV}$. The $\Delta \mathrm{E}-\mathrm{E}$ spectrum on bottom figure shows exclusive ${ }^{26} \mathrm{Al}$ events produced from one of the targets irradiated with deuterium.

showing promising results till this moment; for the second and third cases we obtained total reaction cross sections which are in a good agreement with other data found in the literature. The case of the ${ }^{10} \mathrm{Be}$ reaction is special, considering the reaction studied was not directly measured in previous studies, showing thus, the importance of the experimental method used in this work.

For further studies, and once we proved the effectiveness of the method, we plan to obtained the total cross sections for different energies of the ${ }^{28} \mathrm{Si}(\mathrm{d}, \alpha)^{26} \mathrm{Al}$ reaction, as well as to revisit the ${ }^{25} \mathrm{Mg}(\mathrm{p}, \gamma)^{26} \mathrm{Al}$, which was previously studied with the same technique [16] and some others, show-

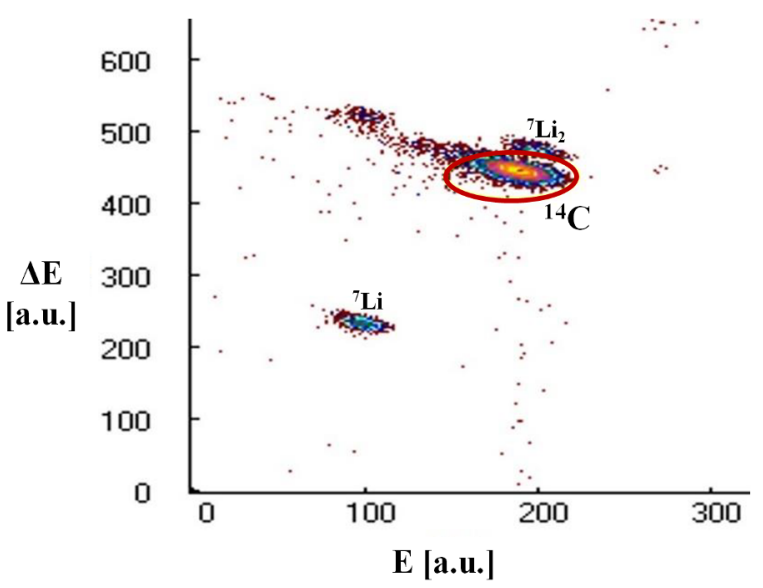

Figure 5. $\Delta \mathrm{E}$-E spectrum coming from uracil target after the neutron irradiation to produce ${ }^{14} \mathrm{C}$. Enclosed in red the ${ }^{14} \mathrm{C}$ events, which are well separated from ${ }^{7} \mathrm{Li}_{2}$ molecule.

ing some discrepancies around $400 \mathrm{keV}$, according to that found in the literature. This last reaction is now possible at LEMA facility thanks to its recent new beam line [26], where intense proton beams at low energies can be produced.

\section{Acknowledgements.}

The authors want to thank the technical support from ININ, Carlos Graef Laboratory and LEMA staffs. This work was partially supported for DGAPA-UNAM IN107820, AG101120 and CONACyT 314857 projects.

\section{References}

[1] L. Csedreki, et. al., Nucl. Inst. Meth. Phys. Res. A 994, 165081 (2021) 


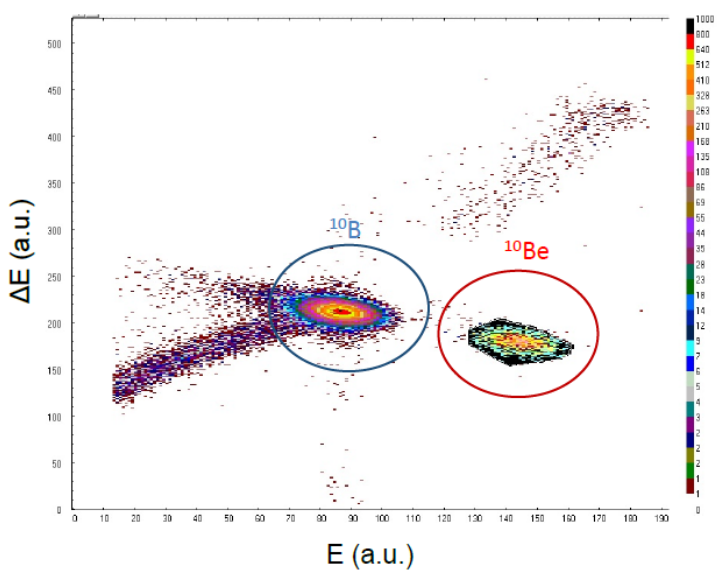

Figure 6. $\Delta \mathrm{E}-\mathrm{E}$ spectrum from $\mathrm{BeO}$ samples irradiated with neutrons to produce ${ }^{10} \mathrm{Be}$. The circles show the well separation between ${ }^{10} \mathrm{Be}$ events and those of its isobar, the ${ }^{10} \mathrm{~B}$.

[2] G.G. Rapisarda, et. al., Front. Astron. Space Sci. 7 589240 (2021)

[3] T. Aumann and C. Bertulani, Progr. Part. Nuclear Physics 112, 103753 (2020)

[4] C. Spitaleri et al., Eur. Phys. J. A 52, 77 (2016)

[5] I. Mardor et. al., Eur. Phys. J A 54, 91 (2018)

[6] I.S. Anderson et. al., Phys. Reports 654, 1 (2016)

[7] I. Dillmann and R. Reifarth JINST 7 C04014 (2012)

[8] R. E. Tribble et. al., Rep. Prog. Phys. 77, 106901 (2014)

[9] A. Best et al., Eur. Phys. J. A 52, 72 (2016)

[10] J. Wagemans, C. Wagemans, G. Goeminne, and P. Geltenbort, Phys. Rev. C 61, 064601 (2000)
[11] S. Mughabghab, Atlas of Neutron Resonances, 5th ed. (Elsevier, Amsterdam, 2006)

[12] C. M. Conneely, W. V. Prestwich, and T. J. Kennett, Nucl. Instrum. Methods Phys. Res. A 248, 416 (1986)

[13] D. D. Clayton, S. A. Colgate, and G. J. Fishman, Astrophys. J. 155, 75 (1969)

[14] T. Lee, D. a. Papanastassiou, and G. J. Wasserburg, Geophys. Res. Lett. 3, 1 (1976)

[15] J. Knödlseder et. al., Astron. and Astrophys. 344 (1999)

[16] A. Arazi et. al., Phys. Rev. C. 74025802 (2006)

[17] E. Andrade, et. al., Nucl. Inst. Meth. Phys. Res. B 332 (2014)

[18] G. Murillo, et. al., Nucl. Inst. Meth. Phys. Res. B 136-138 888-892 (1998)

[19] H. S. Cruz-Galindo and R. Raya-Arredondo, AIP Conf. Proc. 1544, 53 (2013)

[20] C. Solís, E. Chávez, M.E. Ortiz, A. Huerta, E. Andrade, E. Barrios. Nucl. Inst. Meth.Phys. B 331 (2014)

[21] V. Araujo-Escalona, et. al., Phys. Procedia 90, 421-428 (2017)

[22] L. Acosta, et. al., Eur. Phys. J. Web Conf. 165, 01001 (2017)

[23] G. Reza, et. al., Eur. Phys. J. Plus 135, 899 (2020)

[24] D.J. Marín-Lámbarri et. al., Phys. Rev. C 102, 044601 (2020)

[25] K. Nishiizumi, M. Imamura, M.W. Caffee, J. R. Southon, R. C. Finkel, and J. McAninch, Nucl. Instrum. Methods Phys. Res. B 258, 403 (2007)

[26] L. Acosta, et. al., J. Phys. Conf. Ser. 1643, 012029 (2020) 